\title{
DEVELOPMENT OF A THEORETICAL APPROACH TO THE CONDITIONAL OPTIMIZATION OF AIRCRAFT MAINTENANCE PREFERENCE UNCERTAINTY
}

\author{
Andriy GONCHARENKO* \\ National Aviation Universit, Kosmonavta Komarova ave. 1, 03680 Kyiv, Ukraine
}

Received 30 July 2018; accepted 20 September 2018

\begin{abstract}
The paper builds on the ideas of previous research concerning the theoretical explanation of the aircraft operational process with regard to the preferences for maintenance organization by experts and aircraft operators, and describes the designed mathematical models. The problem of conditional extremization is considered. The uncertainty of aircraft technical operation multi-alternativeness is evaluated using the subjective entropy of the aircraft operators' and experts' preferences. By applying the subjective entropy extremization principle in view of its maximum, we obtain the conditional optimal distributions of the preferences. The proposed approach allows finding the optimal distribution of the aircraft fleet for the available maintenance alternatives, taking into consideration the restricted possible influences or shadow components of maintenance organizations. The concepts discussed here are important for evaluating the effectiveness of the aviation industry by making allowance for shadow parameters, if needed. The designed model is illustrated with diagrams.
\end{abstract}

Keywords: aircraft operation, airworthiness support, flight safety, aircraft maintenance, multi-alternativeness, uncertainty, subjective entropy extremization principle, optimization, shadow economy, individual preferences.

\section{Introduction}

It is important to perform proper aircraft maintenance at the aircraft maintenance organizations (AMOs) officially approved by ICAO, EASA, and the National Civil Aviation Authorities (NCAA). The set of the approved AMOs is a set of competitive alternatives available for aircraft operators (AOs) to maintain the AOs' or airlines' aircraft fleets (AAFs).

Probabilistic uncertainty for maintenance processes, as considered in (Dhillon, 2006) and (Nakagawa, 2005), arises together with the uncertainty of the alternative AMOs preferences by AOs. The theoretical aspect of aircraft operation as depending on the uncertainty of the maintenance alternatives individuals' subjective preferences has already been introduces in a preceding publication (Goncharenko, 2017).

A lot of possibilities for aircraft maintenance and repair techniques are described in (Kroes, Watkins, Delp, \& Sterkenburg, 2013), considering the entire aircraft, whereas the study-guide (Wild \& Kroes, 2014) gives a good deal of help for the selection of appropriate technologies for aircraft powerplants. However, a plausible explanation for who, what, how, how much, where, when, etc. will prefer the use of it has not been presented and remains a scientific research gap.

For this purpose, the theory of subjective analysis (Kasianov, 2013; Kasianov, Goncharenko, 2015, 2017; Goncharenko, 2017) allows developing a mathematical model for obtaining the functions of such subjective preferences in an explicit form and substantiated manner.

Many authors across the world emphasize the significance of aviation maintenance quality assessment (Gališanskis, 2004) and the airworthiness challenges that have to be faced in both civil and military aviation (Thian, 2015; Le \& Lappas, 2015). The issues of the available multialternativeness in relation to airworthiness management and aircraft maintenance in fast developing economies of India and China are discussed in a recent publication (Kourousis \& Comer, 2018). There are problems caused by imperfect work performed all over the globe. Once again, the objective uncertainty in probabilistic events as well as the subjective uncertainty in the preferences of the available alternatives have to be taken into scientific consideration in order to predict and simulate the developments in aircraft operational situations.

${ }^{*}$ Corresponding author. E-mail: andygoncharenco@yahoo.com 
Both of these types of uncertainties occur in other fields of aviation activities, for example, airport noise pollution problems (Zaporozhets, Tokarev, \& Attenborough, 2011) or dealing with the multi-alternativeness of unmanned aerial vehicle robust system equipment (Sushchenko \& Goncharenko, 2016).

The sphere of the subjective analysis entropy paradigm (Kasianov, 2013; Kasianov, Goncharenko, 2015, 2017; Goncharenko, 2017) encompasses aspects of training and education of aviation specialists (Kulyk \& Suslova, 2014), decision support in air navigation (Shmelova, Sikirda, Rizun, Salem, \& Kovalyov, 2017) as well as the flight support operational radio systems (Solomentsev, Zaliskyi, \& Zuiev, 2016).

In all the areas mentioned above, there is a necessity for the evaluation and quantitative estimation of a person's who is responsible for the decision making individual subjective preference system distribution in an explicit view. Even future prospective aviation industry enterprises would definitely require an officially approved maintenance system taking into consideration someone's individual preferences uncertainty measure with regard to achievable operational alternatives.

The aim of the present paper is to develop a model of conditional optimization of the AAFs divergence between two AMOs in the framework of the theory of subjective analysis (Kasianov, 2013; Kasianov \& Goncharenko, 2015, 2017; Goncharenko, 2017). That is to obtain mathematical expressions that take into account the AOs' subjective preferences for alternative AMOs in the explicit view, which helps to distribute the AAFs between these two options.

Such an approach implies the use of the subjective entropy maximum principle (SEMP) (Kasianov, 2013). This paper continues the previous research in (Kasianov, 2013; Kasianov \& Goncharenko, 2015, 2017; Goncharenko, 2017).

According to the aim, the improved theoretical approach includes the explanation of the aircraft operational process with regard to the maintenance organizations preferences constrained to the AMOs resources. This is a kind of an isoperimetric condition.

Thus, theoretically, the problem setting proposed in the present paper will require one more, additional, extra, constrain (the restriction or limitation of the AMOs resources influence on the required decision making process result) as compared to the previous research conducted in Goncharenko (2017), where only the normalizing condition constrain has been taken into account.

\section{Conditional optimization}

A significant part of this problem setting is the consideration of conditional optimality.

It is obvious that the AMOs resources used to achieve the needed impact on the AOs and experts are restricted. It is quite logical to presume that the measured influence of the AMO on the experts has a limitation.
When expressing the arguments of the shadow contributions, $\left(C_{1}^{I I}\right.$ is the special utility/effectiveness/cognitive dimensionless (possibly ratio) function, allowing mathematically logarithmic operation (either a score or even a contribution or shadow financial component), which has been obtained by the second AMO in the first expert's opinion; $C_{1}^{I}$ is the corresponding cognitive function of the first inspector for the first AMO; $C_{2}^{I}$, and $C_{2}^{I I}$ are the corresponding contributions of the first and second AMO to the cognitive functions of the second inspector), for example:

$$
C_{2}^{I}=\Sigma^{I}-C_{1}^{I} ; C_{2}^{I I}=\Sigma^{I I}-C_{1}^{I I},
$$

where $\Sigma^{I}$ and $\Sigma^{I I}$ are the total amounts of the corresponding AMOs' resources granted to persuade both inspectors. We obtain the whole problem setting based on the conditions of Equation (1) with just two independent variables, i.e. $C_{1}^{I}$ and $C_{1}^{I I}$.

For instance, the second expert's preference distribution is obtained from a functional as well as from the operational purpose functional (Kasianov, 2013; Kasianov \& Goncharenko, 2015, 2017; Goncharenko, 2017, Eq. (8)). Here the operational purpose functional will be:

$$
\begin{aligned}
& \Phi_{\pi_{2}}=-\pi_{2}^{I} \ln \pi_{2}^{I}-\pi_{2}^{I I} \ln \pi_{2}^{I I}+\beta \pi_{2}^{I} \ln \left[C_{2}^{I}\left(C_{1}^{I}\right)\right]+ \\
& \beta \pi_{2}^{I I} \ln \left[C_{2}^{I I}\left(C_{1}^{I I}\right)\right]+\gamma\left(\pi_{2}^{I}+\pi_{2}^{I I}-1\right),
\end{aligned}
$$

where $\Phi_{\pi_{2}}$ is the operational functional value extremized by the second expert on the set of the two reachable (achievable) alternatives of the possible AMOs; $\pi_{2}^{I}$ is the preference of the first AMO by the second inspector; $\pi_{2}^{I I}$ is the preference of the second inspector for the second $\mathrm{AMO} ; \beta$ is the endogenous parameter of the inspector's psych; $\gamma$ is the Lagrange multiplier.

In Equation (2), the member of:

$$
-\pi_{2}^{I} \ln \pi_{2}^{I}-\pi_{2}^{I I} \ln \pi_{2}^{I I}
$$

is the entropy (measure of uncertainty) of the second inspector's preferences with regard to the two AMOs (the subscripts "2" and superscripts " $I$ " and "II" by the preference $\pi$ designation). In the cognitive function, effectiveness function, the member of:

$$
\beta \pi_{2}^{I} \ln C_{2}^{I}(\cdot)+\beta \pi_{2}^{I I} \ln C_{2}^{I I}(\cdot) .
$$

$\beta>0$, because the ideology of the operational purpose functional in Equation (2) implies a raise of the given AMO's preference if the AMO's value increases, i.e. the second inspector prefers the first AMO if he/she gains more use from the first AMO (in the logarithmic scale), the same with the second AMO; the member of:

$$
\left(\pi_{2}^{I}+\pi_{2}^{I I}-1\right)
$$

is the normalizing condition for the second inspector's preferences for the operational alternatives of AMOs.

In accordance with the SEMP, the preferences distributions in the so-called canonical view are obtained with the use of the necessary conditions for the extremum existence in Equation (2):

$$
\frac{\partial \Phi_{\pi_{2}}}{\partial \pi_{2}^{I}}=-\ln \pi_{2}^{I}-1+\beta \ln C_{2}^{I}(\cdot)+\gamma=0 .
$$


By applying the same condition for the second AMO's preference by the second expert as in Equation (6) and using the normalizing condition in Equation (5), it yields the sought extremal canonical view distribution for the preferences:

$$
\begin{aligned}
& \pi_{2}^{I}\left(C_{1}^{I}, C_{1}^{I I}\right)=\frac{\left[C_{2}^{I}\left(C_{1}^{I}\right)\right]^{\beta}}{\left[C_{2}^{I}\left(C_{1}^{I}\right)\right]^{\beta}+\left[C_{2}^{I I}\left(C_{1}^{I I}\right)\right]^{\beta}}= \\
& \frac{\left(\Sigma^{I}-C_{1}^{I}\right)^{\beta}}{\left(\Sigma^{I}-C_{1}^{I}\right)^{\beta}+\left(\Sigma^{I I}-C_{1}^{I I}\right)^{\beta}} ; \\
& \pi_{2}^{I I}\left(C_{1}^{I}, C_{1}^{I I}\right)=\frac{\left(\Sigma^{I I}-C_{1}^{I I}\right)^{\beta}}{\left(\Sigma^{I}-C_{1}^{I}\right)^{\beta}+\left(\Sigma^{I I}-C_{1}^{I I}\right)^{\beta}} .
\end{aligned}
$$

Then, the AO's preferences are obtained from a functional, similar to the operational purpose functional (Kasianov, 2013; Kasianov, Goncharenko, 2015, 2017; Goncharenko, 2017, Eq. (9)):

$$
\begin{aligned}
& \Phi_{\pi_{A O}}=-\pi_{I} \ln \pi_{I}-\pi_{I I} \ln \pi_{I I}+ \\
& \beta \pi_{I}\left[F_{I} \pi_{1}^{I}\left(C_{1}^{I}, C_{1}^{I I}\right) \pi_{2}^{I}\left(C_{1}^{I}, C_{1}^{I I}\right)\right]+ \\
& \beta \pi_{I I}\left[F_{I I} \pi_{1}^{I I}\left(C_{1}^{I}, C_{1}^{I I}\right) \pi_{2}^{I I}\left(C_{1}^{I}, C_{1}^{I I}\right)\right]+ \\
& \gamma\left(\pi_{I}+\pi_{I I}-1\right),
\end{aligned}
$$

where $\Phi_{\pi_{A O}}$ is the operational purpose functional value optimized (extremized) by the AO on the set of the two reachable (achievable) alternatives for him/her of the possible AMOs; $\pi_{I}$ is the preference of the first AMO; $\pi_{I I}$ is the preference of the second AMO; $\beta$ is the endogenous parameter of the AO's psych (it is deemed unchanged by its absolute value $|\beta|$ as of the inspectors', which also presumably simplifies the problem setting, although in Equation (8), with the same module $|\beta|$, it is also a positive value, i.e. $\beta>0) ; F_{I}$ and $F_{I I}$ are the cognitive functions (utility/score) obtained by the first and second AMO in the AO's opinion respectively; $\pi_{1}^{I}$ and $\pi_{1}^{I I}$ are the corresponding preferences of the first inspector for the AMOs.

$$
\begin{aligned}
& \pi_{I}\left(C_{1}^{I}, C_{1}^{I I}\right)=\frac{e^{\beta\left(F_{I} \pi_{1}^{I} \pi_{2}^{I}\right)}}{e^{\beta\left(F_{I} \pi_{1}^{I} \pi_{2}^{I}\right)}+e^{\beta\left(F_{I I} \pi_{1}^{I I} \pi_{2}^{I I}\right)}} ; \\
& \pi_{I I}\left(C_{1}^{I}, C_{1}^{I I}\right)=\frac{e^{\beta\left(F_{I I} \pi_{1}^{I I} \pi_{2}^{I I}\right)}}{e^{\beta\left(F_{I} \pi_{1}^{I} \pi_{2}^{I}\right)}+e^{\beta\left(F_{I I} \pi_{1}^{I I} \pi_{2}^{I I}\right)}} .
\end{aligned}
$$

Now, it is necessary to note that in the case of Equations (2)-(9), the $\beta>0$, because each player, the experts (representatives of ICAO, EASA, and NCAA) and AOs, seeks the maximum benefit for himself/herself and the AAFs are divided between the AMOs in accordance with the preferences distributions.

The necessary conditions for the preferences extremums for the first of the Equations of (9) are:

$$
\begin{aligned}
& \frac{\partial \pi_{I}\left(C_{1}^{I}, C_{1}^{I I}\right)}{\partial C_{1}^{I}}=\left(\frac{\partial \pi_{I}}{\partial C_{1}^{I}}\right)+\frac{\partial \pi_{I}}{\partial \beta} \frac{\partial \beta}{\partial C_{1}^{I}}+ \\
& \frac{\partial \pi_{I}}{\partial F_{I}} \frac{\partial F_{I}}{\partial C_{1}^{I}}+\frac{\partial \pi_{I}}{\partial F_{I I}} \frac{\partial F_{I I}}{\partial C_{1}^{I}}+\frac{\partial \pi_{I}}{\partial \pi_{1}^{I}} \frac{\partial \pi_{1}^{I}}{\partial C_{1}^{I}}+ \\
& \frac{\partial \pi_{I}}{\partial \pi_{1}^{I I}} \frac{\partial \pi_{1}^{I I}}{\partial C_{1}^{I}}+\frac{\partial \pi_{I}}{\partial \pi_{2}^{I}} \frac{\partial \pi_{2}^{I}}{\partial C_{1}^{I}}+\frac{\partial \pi_{I}}{\partial \pi_{2}^{I I}} \frac{\partial \pi_{2}^{I I}}{\partial C_{1}^{I}}=0 . \\
& \text { Here, in Equation (10) } \frac{\partial \pi_{I}\left(C_{1}^{I}, C_{1}^{I I}\right)}{\partial C_{1}^{I}} ; \frac{\partial \beta}{\partial C_{1}^{I}} ; \frac{\partial F_{I}}{\partial C_{1}^{I}} ;
\end{aligned}
$$

$\frac{\partial F_{I I}}{\partial C_{1}^{I}} ; \frac{\partial \pi_{1}^{I}}{\partial C_{1}^{I}} ; \frac{\partial \pi_{1}^{I I}}{\partial C_{1}^{I}} ; \frac{\partial \pi_{2}^{I}}{\partial C_{1}^{I}} ; \frac{\partial \pi_{2}^{I I}}{\partial C_{1}^{I}}$ are partial but complete derivatives and $\left(\frac{\partial \pi_{I}}{\partial C_{1}^{I}}\right) ; \frac{\partial \pi_{I}}{\partial \beta} ; \frac{\partial \pi_{I}}{\partial F_{I}} ; \frac{\partial \pi_{I}}{\partial F_{I I}} ; \frac{\partial \pi_{I}}{\partial \pi_{1}^{I}} ; \frac{\partial \pi_{I}}{\partial \pi_{1}^{I I}}$; $\frac{\partial \pi_{I}}{\partial \pi_{2}^{I}} ; \frac{\partial \pi_{I}}{\partial \pi_{2}^{I I}}$ are partial derivatives with respect to their arguments.

\section{Results}

Since the first of the Equations of (9) does not depend on $C_{1}^{I}$ in an explicit way and the functions of $\beta, F_{I}$, and $F_{I I}$ do not depend upon it at all, in the considered problem setting, then

$$
\left(\frac{\partial \pi_{I}}{\partial C_{1}^{I}}\right)=\frac{\partial \beta}{\partial C_{1}^{I}}=\frac{\partial F_{I}}{\partial C_{1}^{I}}=\frac{\partial F_{I I}}{\partial C_{1}^{I}}=0 .
$$

Because of that condition in Equation (11),

$$
\frac{\partial \pi_{I}}{\partial \beta} \frac{\partial \beta}{\partial C_{1}^{I}}=\frac{\partial \pi_{I}}{\partial F_{I}} \frac{\partial F_{I}}{\partial C_{1}^{I}}=\frac{\partial \pi_{I}}{\partial F_{I I}} \frac{\partial F_{I I}}{\partial C_{1}^{I}}=0 .
$$

By substituting Equations (11) and (12) for their values into Equation (10), instead we get:

$$
\begin{aligned}
& \frac{\partial \pi_{I}}{\partial C_{1}^{I}}=\frac{\partial \pi_{I}}{\partial \pi_{1}^{I}} \frac{\partial \pi_{1}^{I}}{\partial C_{1}^{I}}+\frac{\partial \pi_{I}}{\partial \pi_{1}^{I I}} \frac{\partial \pi_{1}^{I I}}{\partial C_{1}^{I}}+\frac{\partial \pi_{I}}{\partial \pi_{2}^{I}} \frac{\partial \pi_{2}^{I}}{\partial C_{1}^{I}}+ \\
& \frac{\partial \pi_{I}}{\partial \pi_{2}^{I I}} \frac{\partial \pi_{2}^{I I}}{\partial C_{1}^{I}}=0 . \\
& \frac{\partial \pi_{I}}{\partial \pi_{1}^{I}}=\frac{\beta\left(F_{I} \pi_{2}^{I}\right) e^{\beta\left(F_{I} \pi_{1}^{I I} \pi_{2}^{I}+F_{I I} \pi_{1}^{I I} \pi_{2}^{I I}\right)}}{\left(e^{\beta F_{I} \pi_{1}^{I} \pi_{2}^{I}}+e^{\beta F_{I I} \pi_{1}^{I I} \pi_{2}^{I I}}\right)^{2}} ; \\
& \frac{\partial \pi_{I}}{\partial \pi_{1}^{I I}}=\frac{-\beta\left(F_{I I} \pi_{2}^{I I}\right) e^{\beta\left(F_{I} \pi_{1}^{I} \pi_{2}^{I}+F_{I I} \pi_{1}^{I I} \pi_{2}^{I I}\right)}}{\left(e^{\beta\left(F_{I} \pi_{1}^{I} \pi_{2}^{I}\right)}+e^{\beta\left(F_{I I} \pi_{1}^{I I} \pi_{2}^{I I}\right)}\right)^{2}} ; \\
& \frac{\partial \pi_{I}}{\partial \pi_{2}^{I}}=\frac{\beta\left(F_{I} \pi_{1}^{I}\right) e^{\beta\left(F_{I} \pi_{1}^{I} \pi_{2}^{I}+F_{I I} \pi_{1}^{I I} \pi_{2}^{I I}\right)}}{\left(e^{\beta\left(F_{I} \pi_{1}^{I} \pi_{2}^{I}\right)}+e^{\beta\left(F_{I I} \pi_{1}^{I I} \pi_{2}^{I I}\right)}\right)^{2}} ; \\
& \frac{\partial \pi_{I}}{\partial \pi_{2}^{I I}}=\frac{-\beta\left(F_{I I} \pi_{1}^{I I}\right) e^{\beta\left(F_{I} \pi_{1}^{I} \pi_{2}^{I}+F_{I I} \pi_{1}^{I I} \pi_{2}^{I I}\right)}}{\left(e^{\beta\left(F_{I} \pi_{1}^{I} \pi_{2}^{I}\right)}+e^{\beta\left(F_{I I} \pi_{1}^{I I} \pi_{2}^{I I}\right)}\right)^{2}} .
\end{aligned}
$$


After the use of the operational purpose functional (Kasianov, 2013; Kasianov, Goncharenko, 2015, 2017; Goncharenko, 2017, Eq. (8)):

$$
\begin{aligned}
& \Phi_{\pi_{1}}=-\pi_{1}^{I} \ln \pi_{1}^{I}-\pi_{1}^{I I} \ln \pi_{1}^{I I}+\beta \pi_{1}^{I} \ln C_{1}^{I}+ \\
& \beta \pi_{1}^{I I} \ln C_{1}^{I I}+\gamma\left(\pi_{1}^{I}+\pi_{1}^{I I}-1\right) ; \\
& \frac{\partial \pi_{1}^{I}}{\partial C_{1}^{I}}=\frac{\beta\left(C_{1}^{I}\right)^{\beta-1}\left(C_{1}^{I I}\right)^{\beta}}{\left[\left(C_{1}^{I}\right)^{\beta}+\left(C_{1}^{I I}\right)^{\beta}\right]^{2}} ; \\
& \frac{\partial \pi_{1}^{I I}}{\partial C_{1}^{I}}=\frac{-\beta\left(C_{1}^{I I}\right)^{\beta}\left[\left(C_{1}^{I}\right)^{\beta-1}\right]}{\left[\left(C_{1}^{I}\right)^{\beta}+\left(C_{1}^{I I}\right)^{\beta}\right]^{2}} .
\end{aligned}
$$

From Equations (2) and (7), it follows that:

$$
\begin{gathered}
\frac{\partial \pi_{2}^{I}}{\partial C_{1}^{I}}=\frac{-\beta\left(\Sigma^{I}-C_{1}^{I}\right)^{\beta-1}\left(\Sigma^{I I}-C_{1}^{I I}\right)^{\beta}}{\left[\left(\Sigma^{I}-C_{1}^{I}\right)^{\beta}+\left(\Sigma^{I I}-C_{1}^{I I}\right)^{\beta}\right]^{2}} ; \\
\frac{\partial \pi_{2}^{I I}}{\partial C_{1}^{I}}=\frac{+\beta\left(\Sigma^{I I}-C_{1}^{I I}\right)^{\beta}\left[\left(\Sigma^{I}-C_{1}^{I}\right)^{\beta-1}\right]}{\left[\left(\Sigma^{I}-C_{1}^{I}\right)^{\beta}+\left(\Sigma^{I I}-C_{1}^{I I}\right)^{\beta}\right]^{2}} .
\end{gathered}
$$

By inserting Equations (14), (16), and (17), into Equation (13), we get the expression:

$$
\begin{gathered}
\frac{\beta^{2} e^{\beta\left(F_{I} \pi_{1}^{I} \pi_{2}^{I}+F_{I I} \pi_{1}^{I I} \pi_{2}^{I I}\right)}}{\left(e^{\beta F_{I} \pi_{1}^{I} \pi_{2}^{I}}+e^{\beta F_{I I} \pi_{1}^{I I} \pi_{2}^{I I}}\right)^{2}}\left(\frac{\left(C_{1}^{I}\right)^{\beta-1}\left(C_{1}^{I I}\right)^{\beta}\left(\begin{array}{l}
F_{I} \pi_{2}^{I}+ \\
F_{I I} \pi_{2}^{I I}
\end{array}\right)}{\left[\left(C_{1}^{I}\right)^{\beta}+\left(C_{1}^{I I}\right)^{\beta}\right]^{2}}-\right. \\
\left.\frac{\left(F_{I} \pi_{1}^{I}+F_{I I} \pi_{1}^{I I}\right)\left(\Sigma^{I}-C_{1}^{I}\right)^{\beta-1}\left(\Sigma^{I I}-C_{1}^{I I}\right)^{\beta}}{\left[\left(\Sigma^{I}-C_{1}^{I}\right)^{\beta}+\left(\Sigma^{I I}-C_{1}^{I I}\right)^{\beta}\right]^{2}}\right)=0 .
\end{gathered}
$$

The results of the modelling, for example, for the first inspector's preference functions obtained as the solution of the conditional optimization of the functional Equation (15), are shown in Figures 1 and 2.

When illustrating the application of the results of the derivation of the analytic solution to the considered objective functional, it should be mentioned that the theoretical contemplations of the present paper are centered at the principle dependences. Therefore, some simplifications with allowable values of fictitious parameters seem to be appropriate.

Consider the $\beta=2$ parameter value, illustrated in the example shown in Figures 1 and 2.

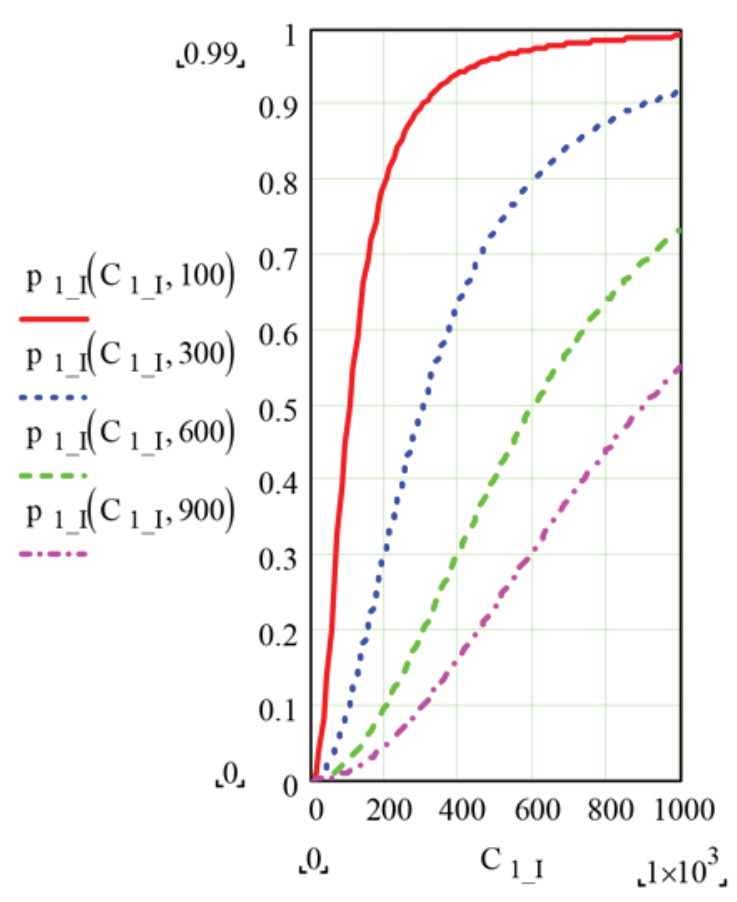

Figure 1. Changes of the first inspector's preferences for the first maintenance organization

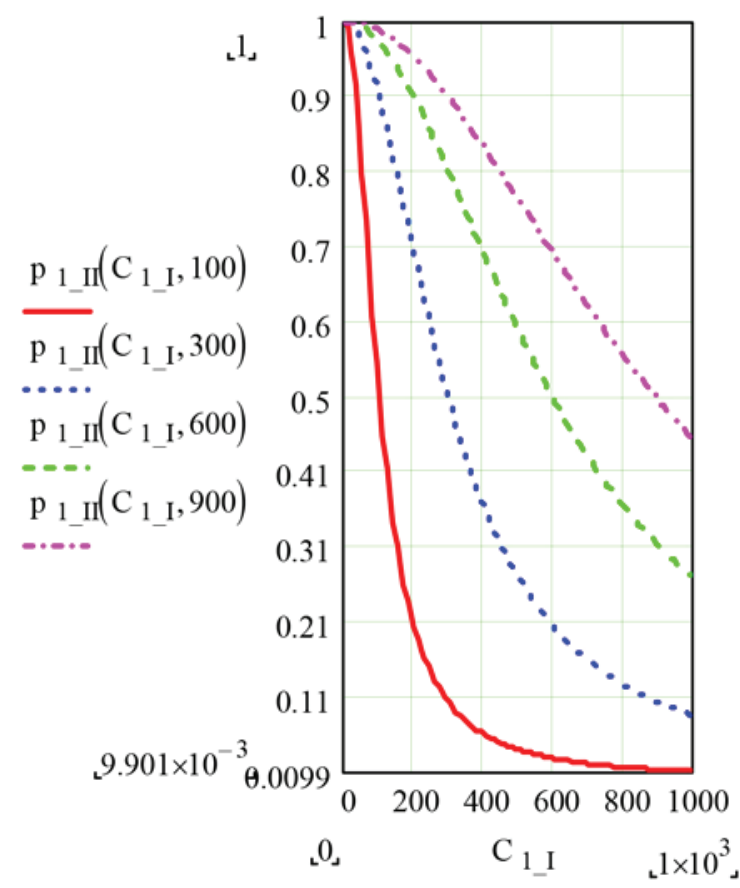

Figure 2. Changes of the first inspector's preferences for the second maintenance organization

\section{Discussion}

In Figures 1 and 2 , the designation of $\mathrm{p}_{1_{-} \mathrm{I}}\left(\mathrm{C}_{1_{-} \mathrm{I}}, \ldots\right)$ stands for the first inspector's preferences functions obtained as a solution of the conditional optimization $\pi_{1}^{I}(\cdot)$ of the functional Equation (15); $C_{1 \_I}$ stands for $C_{1}^{I}$, and $C_{1}^{I I}$ takes the values of 100; 300; 600; and 900 respectively; $\mathrm{p}_{1 \_I I}\left(\mathrm{C}_{1 \_\mathrm{I}}, \ldots\right)$ depicts $\pi_{1}^{I I}(\cdot)$. 
It is easier to analyse Equation (18) with some simplified assumptions:

$$
\beta=F_{I}=F_{I I}=1 \text {. }
$$

Then, under the condition of Equation (19), the expression of Equation (18) yields:

$$
\begin{aligned}
& C_{1}^{I I}\left(\Sigma^{I}+\Sigma^{I I}-C_{1}^{I I}-C_{1}^{I}\right)^{2}- \\
& \left(C_{1}^{I}+C_{1}^{I I}\right)^{2}\left(\Sigma^{I I}-C_{1}^{I I}\right)=0 .
\end{aligned}
$$

From Equation (20), we obtain:

$$
\begin{aligned}
& \left(2 C_{1}^{I I}-\Sigma^{I I}\right)\left(C_{1}^{I}\right)^{2}-2 C_{1}^{I I}\left[\Sigma^{I}+2\left(\Sigma^{I I}-C_{1}^{I I}\right)\right] C_{1}^{I}+ \\
& C_{1}^{I I}\left[\left(\Sigma^{I}+\Sigma^{I I}-C_{1}^{I I}\right)^{2}-\left(\Sigma^{I I}-C_{1}^{I I}\right)\left(C_{1}^{I I}\right)\right]=0 .
\end{aligned}
$$

The roots of Equation (21) are the optimal values of $C_{1}^{I}$, the optimal influence of the first AMO, exerted upon the first inspector, aimed at gaining the most effect from the AO, that is to draw its AAF, i.e.

$$
\left(C_{1}^{I}\right)_{1,2}^{o p t}=\frac{-b \pm \sqrt{b^{2}-4 a c}}{2 a}
$$

where the coefficients from Equations (21) and (22) are:

$$
\begin{aligned}
& b=-2 C_{1}^{I I}\left[\Sigma^{I}+2\left(\Sigma^{I I}-C_{1}^{I I}\right)\right] ; a=\left(2 C_{1}^{I I}-\Sigma^{I I}\right) ; \\
& c=C_{1}^{I I}\left[\left(\Sigma^{I}+\Sigma^{I I}-C_{1}^{I I}\right)^{2}-C_{1}^{I I}\left(\Sigma^{I I}-C_{1}^{I I}\right)\right] .
\end{aligned}
$$

Hence, at some given limitation ranges of $\Sigma^{I}$ and $\Sigma^{I I}$, as well as with the conditions of Equations (1)-(21), also with a fixed value of $C_{1}^{I I}$, the first AMO can optimally draw the AO's attention by means of an optimal impact on the first inspector $\left(C_{1}^{I}\right)_{1,2}^{o p t}$, Equation (22).

The importance of the solutions (see Figures 1 and 2) is that the concepts under discussion are significant for the evaluation of the aviation industry's effectiveness, making allowance for shadow parameters, if needed.

It should be pointed out that explicitly expressed preferences about the AAFs distribution between the two available AMOs, in all of the considered areas or spheres of the decision making, give the AOs a powerful tool to manage the alternatives beneficially, depending on a specific practical scenario. Such a task was stated and emphasized in the introduction section of the paper.

The model constructed here, as mentioned above, develops the ideas of Goncharenko (2017), by taking into account not only the normalizing condition for preferences similar to Eq. (5), but also the influences' constraints in view of Eq. (1) with the conditional optimization of the objective functionals (2), (8) or (15), with respect to the preferences degree of uncertainty (3).

Under such conditions, the entropies of the experts' preferences, in view of expressions like (3), become mutually dependable via the two independent variables $C_{1}^{I}$ and $C_{1}^{I I}$ (see Figures 1 and 2), as well as constraint (1). Therefore, this allows using more adequate modelling for aircraft technical operation, maintenance, and airworthiness support.

\section{Conclusions}

The proposed approach of SEMP application, is a convenient tool for modelling the behaviour of an active AO.
Further research should focus on a more detailed simulation in order to formulate a prognosis, based on conditional optimization and the theoretical speculations expressed with Equations (1)-(23).

\section{References}

Dhillon, B. S. (2006). Maintainability, maintenance, and reliability for engineers. New York: Taylor \& Francis Group. https://doi.org/10.1201/9781420006780

Gališanskis, A. (2004). Aspects of quality evaluation in aviation maintenance. Aviation, 8(3), 18-26.

Goncharenko, A. (2017). Aircraft operation depending upon the uncertainty of maintenance alternatives. Aviation, 21(4), 126131. https://doi.org/10.3846/16487788.2017.1415227

Kasianov, V. (2013). Subjective entropy of preferences. Subjective analysis. Warsaw, Poland: Institute of Aviation Scientific Publications. Retrieved from http://kasianovv.wixsite.com/ entropyofpreferences

Kasianov, V. A., \& Goncharenko, A. V. (2015). Variational principles of subjective analysis. Modified Euler-Lagrange variational principle. Entropy approach. Kyiv, Ukraine: SE TPC "Pryoritety”. Retrieved from http://media.wix.com/ugd/4dca65_c80b9fde57744e018ada21ecd32ca678.pdf

Kasianov, V. A., \& Goncharenko, A. V. (2017). Extremal principle of subjective analysis. Light and Shadow. Proportions of shadow economy. Entropy approach. Kyiv, Ukraine: "Kafedra".

Kourousis, K. I., \& Comer, A. (2018). Indian and Chinese aviation industry: the EASA framework option. Aircraft Engineering and Aerospace Technology, 90(2), 246-250.

https://doi.org/10.1108/AEAT-03-2017-0083

Kroes, M. J., Watkins, W. A., Delp, F., \& Sterkenburg, R. (2013). Aircraft maintenance and repair. New York, NY, USA: McGraw-Hill, Education.

Kulyk, M., \& Suslova, G. (2014). Integration of the ICAO Training Institute into the international education network. Aviation, 18(2), 104-108. https://doi.org/10.3846/16487788.2014.926643

Le, H., \& Lappas, I. (2015). Continuing airworthiness: major drivers and challenges in civil and military aviation. Aviation, 19(4), 165-170. https://doi.org/10.3846/16487788.2015.1126909

Nakagawa, T. (2005). Maintenance theory of reliability. London: Springer-Verlag.

Shmelova, T., Sikirda, Y., Rizun, N., Salem, A. B. M., \& Kovalyov, Y. N. (2017). Socio-technical decision support in air navigation systems: emerging research and opportunities. Pennsylvania, USA: International Publisher of Progressive Information Science and Technology Research.

Solomentsev, O., Zaliskyi, M., \& Zuiev, O. (2016). Estimation of quality parameters in the radio flight support operational system. Aviation, 20(3), 123-128.

https://doi.org/10.3846/16487788.2016.1227541

Sushchenko, O., \& Goncharenko, A. (2016). Design of robust systems for stabilization of unmanned aerial vehicle equipment. International Journal of Aerospace Engineering, 2016(2016), Article ID 6054081, 10 pages. https://doi.org/10.1155/2016/6054081

Thian, C. V. (2015). Civil and military airworthiness challenges in Asia. Aviation, 19(2), 78-82. https://doi.org/10.3846/16487788.2015.1057993

Wild, T. W., \& Kroes, M. J. (2014). Aircraft powerplants. New York, NY, USA: McGraw-Hill, Education.

Zaporozhets, O., Tokarev, V., \& Attenborough, K. (2011). Aircraft noise. Assessment, prediction and control. Glyph International, Taylor \& Francis. https://doi.org/10.1201/b12545 